\title{
Earthquake Analyzer using Prediction Commands
}

\author{
Abhishek Singh, Sarthak Bansal, Madhav Chaturvedi
}

\begin{abstract}
Destructive earthquakes usually causes gargantuan casualties. So, to cut back these inimical casualties' analysis are made to reduce despicable and forlorn impacts which they left upon others to just ponder and become lugubrious. These factors measure the decisive casualties it brings and also earthquake and therefore the development of rational prediction model to casualties become a crucial analysis topic, as a result of quality and cognitive content of gift prediction methodology of price, an additional correct prediction model is mentioned by gray correlation theory and BP neural networks. The earthquake can be analyzed succinct by using various technique mainly predictive commands to marshal all the calculated time and magnitude of a potential earthquake have been the topic of the many studies varied ways are tried mistreatment several input variables like temperature exorable, seismic movements and particularly the variable climatic conditions. The relation between recorded seismal-acoustic information associate degreed occurring an abnormal seismic process (ASP). However, it's obstreperous to predict all parameters the placement, time and magnitude of the earthquake by mistreatment this information. This model description is different from others as with the help of the prediction commands most of the paragons and domains are identified and tend to explore the activity of serious Earthquakes. We use the preemptive data information which is collected around the planet. We retrieved the data to perceive that associate degree earthquake reaches the class of exceeds a grade range of eight on Richter Scale. The two main affected areas are in the field of Data Exploration and Data Mapping. Number of occurrences of an earthquake with different magnitude ranges, severity of an earthquake. Mapping is thereby crucial to identify highly affected areas based on Magnitude and Correlation between depth and magnitude. So, based on the above explorations we have made the following predictions. Predictions Magnitude based on depth. Magnitude based on Latitude and Longitude. Depth based on Latitude and Longitude The primitive algorithm used here are the Machine Learning Algorithm I.e. Linear Regression and $\mathrm{K}$ Means Clustering. Firstly, we have made all the predictions via Linear Regression and made different clusters of the Earthquakes which belong to the same subdivision as that of Magnitude or Depth.
\end{abstract}

\section{INTRODUCTION}

Earthquake is inferred as a surfeit of disaster which causes hackneyed impact across the places it occurs and takes

Revised Manuscript Received on September 15, 2020.

* Correspondence Author

Abhishek Singh*, Undergraduate Student, SRM Institute of Science and Technology, Chennai (Tamil Nadu), India.

Sarthak Bansal, Undergraduate Student, SRM Institute of Science and Technology, Chennai (Tamil Nadu), India.

Madhav Chaturvedi, Undergraduate Student, SRM Institute of Science and Technology, Chennai (Tamil Nadu), India.

(C) The Authors. Published by Blue Eyes Intelligence Engineering and Sciences Publication (BEIESP). This is an open access article under the CC BY-NC-ND license (http://creativecommons.org/licenses/by-nc-nd/4.0/)
Keyword: Data Exploration and Data Mapping.

hundreds of years for humans to overcome the tragic and pathetic condition. It causes suffering all around the regions nearby and thus a non-flippant impact on social and economic development is created. To mitigate the impact of war like destruction all around earthquake warning has been widely used and developed in earthquake-prone countries like the east most country Japan to the west most country USA, and Mexico. Aftermath of 2008 Wenchuan earthquake, China started to build its very own EEW system. Proclivity of chances of earthquake to damage the public facilities and buildings collapse, the secondary disasters will be the emergency rescue work even more difficult. Earthquake emergency (EE) can be referred to as the destructive earthquake or major incident occurred under the tyrannical and authoritarian form of the government, in order to maximize reduce casualties, economic losses and social influence, leader, organized, planned, subpoena, coordinated, around the earthquake this is a series of quick and efficient. Through monitoring' analysis, integration, and intelligence response using domains such as IOT together, promoted to one who has a good coordination ability and control ability of organic whole. Therefore, enables the purpose of wisdom city with bolster of information technologies is required more than ever before which included all facilities, neophytes development of dynamic plot for system earthquake emergency response to deduce the real-time information display with radio frequency identification , tracking dynamically, synchronous update and timely feedback to improve the earthquake emergency command disposal ability and the comprehensive efficiency of earthquake rescue is hegemony for its usage.

\section{WORKING}

Statistically, a regression is the technique used for the conversion of magnitude over different magnitude and are of critical importance since any bias data is introduced in statistically analysis applied to data to determine the degree of relation of dependent variable with non-dependent variables. It is the linear method to model the link between a scalar response and instructive variables. The instructive case first variable is termed in as straightforward regression. For over one instructive variable, the ameliorate method is termed multiple regressions. In regression, the relationships square measure shapely mistreatment linear predictor functions whose unknown model parameters square measure calculable from the information. Primary multivariate analysis studied can be compendium to be used extensively in sensible applications. This is as a result of models that rely linearly on their unknown parameters square measure is adaptable than rest of models that square measure non-linearly associated

Published By:

Blue Eyes Intelligence Engineering and Sciences Publication

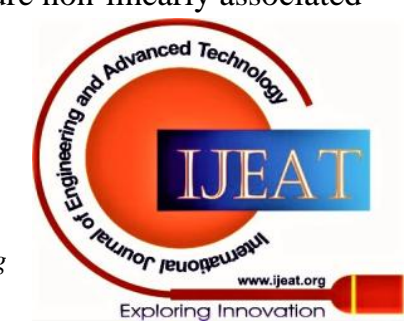




\section{Earthquake Analyzer using Prediction Commands}

with their parameters and since the applied math properties of the ensuing estimator's square measure easier to visualize and observe. Rectilinear regression has plethora of other sensible uses. If the goal is prognostication, or error reduction via method of rectilinear regression may be accustomed work a prophetical model to associate degree discovered knowledge set of values of the response and informative variables. When defining, designing and describing such model, the extra informative variables measures are precariously collected while it is certain that it is not associated to degree incidental to response value, the fitted model may be accustomed to create a prediction of the response. The recapitulated goal, in a nutshell is to clarify vivid displaying within the response variable that attributes to fleeting or flocculation among the data and within the informative variables, rectilinear analysis involves parameters of strength and the link generated between the response especially to see whether or not some informative variables not have any linear relationship with the response relating the minimum of bits .

\section{INFORMATION ACQUISITION AND SIGNAL TRANSMISSION:}

The information and survey regarding signal transmission and analysis is given on the data of earthquakes depends mutually upon the factors or upon the dimensions laid out such as latitude, longitude, magnitude, region and time of the earthquake for the desired region area unit gathered from EMSC. It is required to calculate the time span or gap of the earthquake center at the stations and can give data concerning the relation between magnitude and signal information collected on stations. The system starts to muster or mashal referring to gather, retrieve and store the information from sensors within the stations and sends the data to the desired servers to collect on- site (line) information. The designed signal observation system has a personal attended program that listens to the particular port on a server and records the incoming information to the connected tables of the information. in step with the incoming information which creates a nexus which is connecting the information of two or more data while the system creates the connected table and starts the process of recording. Ramification, no information of acquisition of the data is lost.

\section{ASSUMPTIONS}

Dynamic Plotting is basically classified into plethora of category depending upon the increasing alacrity of identification of system. Basic information management subsystem of city, the thematic map plotting, giving severe commands and dispatching the subsystems. Undermining of basic information management system of city is distributed to map management of the city which are the primitive reasons of creating dynamic plotting of response It basically provide map layout of the entire city, its routes, roads etc. Information management of lifeline engineering is related to people life. It enables management of earthquake system while commanding and dispatching of earthquake responses affected directly so that the given information can be queried easily and it can be refer that information exists or not. Thematic of system plotting system contains automatic plotting, normal plotting, customized mapping and thematic map plot displaying. Earthquake disaster prediction subsystem contains the earthquake disaster management prediction of lifeline engineering, earthquake disaster prediction of building and earthquake disaster prediction the secondary disaster. Aid management system can be described as the info measurement statistical, emergency plus generating and command and dispatching can be enabled. The above assumptions are made to classify dynamic plotting and are classified. The hardware and software system are provided via compatibility and portability using standard models especially rectilinear models with the customary estimation techniques helps in creating a variety of assumptions. Varied extensions are developed that enable assumptions to be relaxed to be taken to consideration a maudlin form or a foible structure and in some cases eliminated completely. The overall structure system depends on ArcGIS secondary development in software side scripting. Application platform is present at the apogee or zenith of the structure. Furthermore, the basic information management and provide compliance service to disaster management. Aid decision are also a part of this thematic map plotting system. It is connected with data platform basic database and information base of lifetime engineering .At the bottom system platform software or hardware platform of the system to ensure safety mechanism of the system. Isometric polynomial commonly called regression or a rectilinear regression. Following are the key assumptions created by customary rectilinear regression models with customary estimation techniques and are remodeled. It is employed as an example in polynomial regression, that uses rectilinear regression to suit the response variable as AN arbitrary polynomial perform a variable quantity. This makes rectilinear regression a particularly powerful illation methodology. Polynomial Regression are puissant meaning powerful in nature and hence can on itself determine the data of magnitude of earthquake , they incline to overfit the info. As a result, solutions setting out of the estimation method. Common examples are Ridge Regression and Lasso Regression. Bayesian rectilinear regression can even be used, that by its nature is additional or less resistant to the matter of overfitting. Banality, ridge regression and lasso regression will each be viewed as special cases of Bayesian rectilinear regression with specific kinds of previous distributions placed on the regression coefficients. To chimerical visualize for error variance, or once a an error is an equally variable round the 'best-fitting line' for all points of $\mathrm{x}$, it's prudent to appear for a "fanning effect" between residual error and foreseen values.. These regression techniques can even be used more than once to find the variance is assumed to be a perform of the mean. Distribution is required instead of standard distribution. This assumes that the errors of the response variables are unrelated to one another. Realistically applied math independence may be a stronger condition than mere lack of correlation and is commonly not required, categorized though as it may be exploited which has a large possibility. Myriad levels of strategies are needed or used to least the number of squares which are capable of handling errors, though they generally need considerably additional knowledge unless some form of regularization is employed to jaundice the model towards assumptive unrelated errors.

Published By:

Blue Eyes Intelligence Engineering and Sciences Publication 
Bayesian rectilinear regression may be a general manner of handling this issue. The generalized linear models, don't suffer from these drawbacks juxtaposing placed these assumptions may actually vary and many different applied math properties of the info powerfully influence the performance of various estimation Methods.

\section{ALGORITHM:}

To begin with the algorithm, it starts with choosing $\mathrm{M}$ random variables as cluster enters defined as centroids. Allocate all rampant variables to the closest of cluster by finding the distance from each centroid of the points. It is required to identify the clusters a new cluster center by taking the average of the assigned points. Again this step is repeated when failed or no data is displayed Now, the next process domicile is that until or unless none of the cluster assignments change it won't affect the opulent of data .Undermining the process until the clusters remain stable, we thereby repeat the algorithm from scratch again as described above.

\section{RESULT AND DISCUSSION}

Since identification of the magnitude of earthquake not only required but at the same time prediction of an earthquake is needed. An analyzing system is needed for making above problem to solve. Earthquake analyzer is a method to do so. Linear Regression and $\mathrm{K}$ means technique are used to solve and analyze the trends of earthquake and thereby predicting the next possibility of earthquake. A graph or a plot can be drawn from the system analyzer and further analyses can be made.

\section{CONCLUSION}

Well , to recapitulate in a nutshell, with our model we can easily get the magnitude and by this it will be highly supportive in analyses and can become maven as by improving the efficiency of deduction of impact of earthquake and henceforth enhancing the performance for many committees to find them and help most of the earthquake-prone areas to stop the disaster and pulverized it. This can give us the brief summary of that is needed to calculate and find the magnitude appropriate manner. The seismic waves get created every day due to different simulations they get worse and can lead to difficult conditions. It can make sure that fewer human lives get across the line and by doing so we can give our best to protect human lives as well as our society.

\section{REFERENCES}

1. T. A. Aliev, A. M. Abbasov, Q. A. Guluyev, F. H. Pashaev, U. E. Sattarova. "System of robust noise monitoring of anomalous seismic processes", Soil Dynamics and Earthquake Engineering, vol:53, pp 11-25, 2013.

2. T.A. Aliev, A.M. Abbasov, E.R. Aliev, G.A. Guluev, "Digital technology and systems for generating and analyzing information from deep strata of the Earth for the purpose of interference monitoring of the technical state of major structures", Automatic Control and Computer Sciences vol: 41, pp: 59-67, 2007.

3. T.A. Aliev, A. M. Alizade, G.D. Etirmishli, G. A. Guluev, F. G. Pashaev, A. G. Rzaev, "Intelligent seismoacoustic system for monitoring the beginning of the anomalous seismic process", Seismic Instruments vol: 47, pp: 27-41, 2011.

\section{AUTHORS PROFILE}

Abhishek Singh, $\mathrm{He}$ is currently pursuing undergraduate degree from SRM Chennai. He will be graduating in the year 2021. His interests are there in the machine learning.

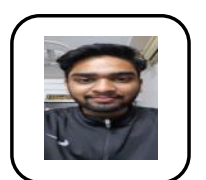

Sarthak Bansal, He is currently pursuing undergraduate degree from SRM Chennai. He will be graduating in the year 2021. His interests are there in the cybersecurity and internet of things.

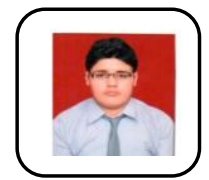

Madhav Chaturvedi, He is currently pursuing undergraduate degree from SRM Chennai. He will be graduating in the year 2021. His interests are there in the cloud computing and data science.

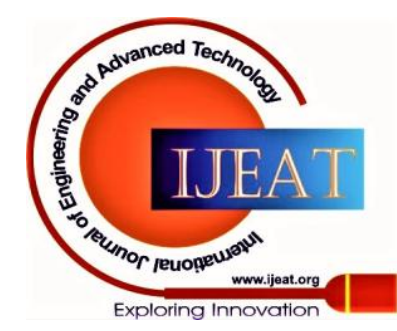

